\title{
EDITORIAL
}

\section{CALIDAD DEL AIRE, PARTÍCULAS EN SUSPENSIÓN Y METALES}

\section{Xavier Querol}

CSIC. Instituto de Diagnóstico Ambiental y Estudios del Agua. Consejo Superior de Investigaciones Científicas. Barcelona.

\section{INTRODUCCIÓN}

La modificación de la composición de la atmósfera por causas antropogénicas o naturales altera la calidad del aire, a la vez que puede causar cambios en el clima por su influencia en el balance radiativo terrestre. Por tanto en lo referente a la perturbación de la composición de la atmósfera se distinguen dos escalas: a) las escalas local, regional y de larga distancia, en las que el deterioro de la calidad del aire o el aporte de determinados contaminantes puede tener repercusiones negativas sobre los ecosistemas, e incluso sobre la salud humana ${ }^{1} ; \mathrm{y}$ b) la escala global, donde el aporte de contaminantes específicos (caso de emisiones de gases de efecto invernadero o de aerosoles atmosféricos) o la destrucción de determinados componentes atmosféricos (caso de la destrucción del ozono estratosférico) pueden modificar el balance radiativo terrestre y por ello inducir cambios en el clima $^{2}$.

El impacto de la contaminación atmosférica sobre la salud es conocido desde antiguo. La momia encontrada en el desierto del Gobi ('The Beauty of Loulans', 1800

\footnotetext{
Correspondencia:

Xavier Querol

Instituto de Diagnóstico Ambiental y Estudios del Agua

Consejo Superior de Investigaciones Científicas.

C/ Jordi Girona 18-26, 08034 Barcelona

Xavier.querol@ija.csic.es
}

antes de nuestra era) se reconoce como la primera evidencia de ello (http://www.co. mendocino.ca.us/aqmd/AQhistory.htm), debido a que los arqueólogos atribuyen su muerte a enfermedades respiratorias causadas por las emisiones de combustión de madera y de polvo mineral. Lao-Tze (año 500 a.C.) reconocía ya el impacto en la calidad del aire de las actividades humanas. Alrededor del año 300 de nuestra era, leyes romanas ya regulaban en York algunas fuentes de contaminación atmosférica, como la producción de cerveza. Alrededor de 1180 Maimónides (Rabi Mose Ben MAIMON, médico sefardí cordobés, 11351204) escribía: Comparar aire de ciudades con el aire de los desiertos y las tierras áridas es como comparar las aguas que son podridas y turbias con las limpias y puras. En la ciudad, a causa de la altura de sus edificios, lo angosto de sus calles y de todo lo que se vierte desde sus habitantes y sus líquidos...el aire se torna estancado, espeso, brumoso y neblinoso...Si el aire se altera alguna vez ligeramente, el estado del Espiritu Psiquico será alterado perceptiblemente.

Pero no solamente existen registros históricos del reconocimiento del impacto de la contaminación antropogénica sobre la calidad del aire, sino también de las emisiones naturales. Así Plinio el Joven describe en una carta a Tácito (VI.16, año 105 d.C.) el deterioro de las funciones respira- 
torias y la posterior muerte de su padre tras el regreso de una visita a Pompeya, el 24 de Agosto del año 79, para observar la erupción del Vesuvio y haber estado expuesto a sus emisiones volcánicas (http://es.wikipedia.org/wiki/Plinio_el_Viejo).

Si bien las causas de la contaminación del aire pueden pues ser naturales (emisiones volcánicas, biogénicas, desérticas, marinas,...) ó antropogénicas, son éstas últimas las que inciden más negativamente sobre la calidad del aire. Existe un gran número de contaminantes atmosféricos con distintas repercusiones en la atmósfera. Entre ellos destacan $\mathrm{CO}_{2}, \mathrm{CO}, \mathrm{SO}_{2}, \mathrm{NO}$, $\mathrm{NO}_{2}, \mathrm{O}_{3}$ (el ozono actúa positivamente en la estratosfera ya que reduce la radiación ultravioleta, pero en la troposfera tiene efectos negativos por su elevado poder oxidante), $\mathrm{NH}_{3}, \mathrm{H}_{2} \mathrm{~S}$, material particulado atmosférico (incluyendo metales, compuestos inorgánicos secundarios y una gran cantidad de compuestos orgánicos, algunos persistentes) y un elevado número de compuestos orgánicos volátiles.

\section{CALIDAD DEL AIRE ACTUAL EN NUESTRAS CIUDADES}

Los indicadores que mejor reflejan la calidad de vida de una sociedad son los medioambientales. Así, solamente cuando estén casi totalmente satisfechas demandas como la asistencia sanitaria, el empleo, la vivienda o el ocio, la protección del medio ambiente ocupa realmente un lugar destacado en la lista de las principales preocupaciones de los ciudadanos.

La sociedad europea demostró una elevada concienciación social en lo concerniente a la contaminación atmosférica y sus efectos adversos sobre la salud en las décadas de 1960 y 1970, desencadenada sobre todo por los episodios de elevada mortalidad de Londres de diciembre de $1952^{8}$. Todo ello condujo a aplicar medidas de reducción progresiva de las emisiones urbanas (como, por ejemplo, la limitación del uso del carbón en calefacciones) y a dar la imagen de que la calidad del aire de las ciudades era ya aceptable. En las últimas décadas se ha producido una focalización del interés social en el cambio climático, más que en la calidad del aire. Sin embargo, las evidencias de causa-efecto probadas por trabajos epidemiológicos de las décadas presente y anterior ${ }^{3-7}$ han demostrado que, incluso a niveles de contaminación relativamente bajos, respecto a los de la década de los setenta existe un impacto significativo de la contaminación en la mortalidad y morbilidad. Ello ha reactivado nuevamente el interés social por este tema y, con ello, la elaboración de nuevas directivas de calidad del aire ${ }^{9,10}$ con objetivos mucho más exigentes que los de las décadas anteriores.

Los principales contaminantes atmosféricos en la Europa más desarrollada son las partículas en suspensión, el dióxido de nitrógeno y el ozono troposférico. Se da el caso de que los valores límite de partículas en suspensión y los óxidos de nitrógeno (Directiva 2008/50/CE ${ }^{10}$ ) se superan principalmente en zonas urbanas, donde habita la mayor parte de la población. Según datos del Ministerio de Medio Ambiente y Medio Rural y Marino, el 60\% de las estaciones de control y vigilancia de la calidad del aire de España situadas en zonas con elevado tráfico rodado superan el valor límite diario de dióxido de nitrógeno fijado para 2010, mientras que el $40 \%$ y el $70 \%$ de ellas superan los valores límite anual y diario de partículas en suspensión fijados ya desde 2005. Es cierto que estos problemas de superación de valores límite de calidad del aire en zonas urbanas presentan una dimensión europea. También es verdad que en la mayor parte de estas zonas el tráfico rodado es la causa principal de las superaciones. Pero, ¿están todas las ciudades europeas igualmente contaminadas? 
Las ciudades escandinavas presentan unos niveles de contaminación claramente inferiores al resto de Europa. Así en las principales zonas urbanas se suelen registrar niveles medios de PM10 (parámetro normativo para partículas en suspensión menores de 10 micras en Europa desde 2001) de alrededor de 20-25 micro-gramos por metro cúbico de aire $\left(\mu \mathrm{g} / \mathrm{m}^{3}\right)$. En ciudades como Berlín, Londres o Ámsterdam dichos niveles suelen aproximarse a 30-35 $\mu \mathrm{g} / \mathrm{m}^{3}$. En Madrid y Barcelona los niveles oscilan entre 35 y $50 \mu \mathrm{g} / \mathrm{m}^{3}$, con los valores superiores registrados en Barcelona. En México DF. éstos varían entre 50 y 70 $\mu \mathrm{g} / \mathrm{m}^{3}$, y en mega-ciudades asiáticas como Pekín o Wuhan, se superan los $125 \mu \mathrm{g} / \mathrm{m}^{3}$. Los niveles de otros contaminantes atmosféricos, aunque con valores de concentración absoluta diferentes, siguen una tendencia similar a la descrita para las partículas en suspensión.

Aunque existen causas meteorológicas que pueden explicar este gradiente, la principal causa son las diferencias en reducción de emisiones contaminantes en las regiones anteriormente consideradas. Desde que en 1999 la CE publicó la Primera Directiva Hija de Calidad del Aire algunos Estados miembros comenzaron a plantearse muy seriamente estrategias de reducción de emisión de contaminantes atmosféricos para alcanzar los niveles de protección de la salud. En base a ello, desde 2003, ciudades como Londres, Berlín, Estocolmo y París, han aplicado una serie de planes y programas en este sentido. Ejemplos son la delimitación de zonas céntricas de baja emisión (en las cuales se reduce drásticamente el tráfico rodado y además se restringe el acceso a los vehículos más contaminantes); peajes para vehículos privados (especialmente a vehículos 4x4) para acceder a zonas céntricas; acciones encaminadas a favorecer el uso del transporte público frente al privado; aplicación de filtros de partículas al transporte público, vehículos de la administración y autobuses escolares; reducción de la velocidad de circulación; aplicación de ordenanzas municipales estrictas para la reducción de las emisiones de la construcción y demolición; favorecer el transporte ferroviario frente al rodado; acciones para favorecer la renovación de parque de vehículos, y una más elevada exigencia a las emisiones industriales, domésticas y residenciales; entre otras.

En las ciudades españolas es importante destacar además el incremento y 'dieselización’ del parque de vehículos. Los vehículos diesel, de la década de los noventa y principios de la presente, sin estar provistos de filtros de partículas y catalizadores específicos para $\mathrm{NO}_{2}$, emiten niveles mucho más elevados de $\mathrm{NO}_{2}$ y partículas en suspensión que otros tipos de vehículos. Actualmente la mayor parte de vehículos diesel ya están equipados con filtros y catalizadores. Además, existen condiciones específicas del clima mediterráneo que propician muy frecuentemente episodios de contaminación. Por ejemplo, la baja precipitación no facilita el lavado de la atmósfera, no elimina el polvo sedimentado sobre la superficie urbana, y por tanto favorece la continua resuspensión del mismo. Características climáticas típicas del Mediterráneo y que son causas de atracción turística (sol, poca lluvia, calmas, orografía intricada) son muy negativas para la calidad del aire, en cuanto que favorecen la acumulación y generación de contaminantes. Por otra parte, la arquitectura urbana de nuestras ciudades con altos edificios, escasas zonas verdes y calles relativamente estrechas favorecen también la acumulación de estos contaminantes.

Considerando todas estas razones, a pesar de los esfuerzos hechos por la industria automovilística para reducir emisiones individuales de los vehículos, los niveles de algunos contaminantes no están disminuyendo en nuestras ciudades ${ }^{11}$. Es más, algunos estudios muestran que posiblemente debido a la 'dieselización' y al incremento 
marcado del parque de vehículos los niveles de partículas sub-micrónicas se han incrementado notablemente en las últimas décadas $^{12,13}$. Así mismo, los niveles de dióxido de nitrógeno se han mantenido o incluso incrementado en el último decenio debido a las mayores tasas de emisión de óxidos de nitrógeno del diesel, y al elevado ratio dióxido de nitrógeno/óxidos de nitrógeno del motor diesel. Por otra parte, la alta emisión derivada del desgaste de frenos y ruedas del incrementado parque de vehículos ha causado que los niveles de metales como cobre, antimonio, estaño, manganeso, zinc y bario registrados en zonas urbanas sean equivalentes, o incluso en algunos casos bastante superiores, a los registrados en zonas industriales $^{14}$.

\section{CONTROL DE LA CALIDAD DEL AIRE}

El objetivo del control de la calidad del aire es doble: a) evaluar la calidad del aire y establecer objetivos que nos permitan, en las sociedades desarrolladas, exigir estrategias (tecnológicas y no tecnológicas) para reducir las emisiones y, por tanto, la exposición de la población a determinados contaminantes; b) investigar las relaciones causa efecto entre los niveles de exposición de la población permite definir umbrales de protección que luego sean utilizados en la normativa ambiental. En el presente volumen, se publican dos artículos ${ }^{15,16}$ referentes a la evaluación de la calidad del aire en las Palmas de Gran Canaria y Santa Cruz de Tenerife el primero, y a los niveles de metales pesados en población general adulta que habita una zona próxima a una planta de valorización energética de residuos en Euskadi el segundo. Ambos tratan problemáticas de gran interés ambiental, de salud pública y científico.

En el primero de los artículos se evalúan los niveles de los principales contaminantes atmosféricos para el periodo 2000 a 2004.
Se destaca que aunque para determinados contaminantes industriales se presentan mayores concentraciones en Santa Cruz, son los óxidos de nitrógeno y las partículas en suspensión los parámetros más problemáticos. En el caso de $\mathrm{NO}_{2}$ se debe a las emisiones del tráfico, y en el de las partículas, además de las emisiones antropogénicas, se destaca la influencia de emisiones de polvo natural desde el cercano continente Africano. Los episodios de 'calima' (causados por las intrusiones de masas de aire africano con elevada carga mineral) inciden marcadamente en la calidad del aire de las Islas Canarias ${ }^{17,18}$ y con menor intensidad sobre la Península ${ }^{19}$ y el archipiélago Balear. Los autores describen también patrones estacionales para los niveles de ozono diferentes a los descritos para muchas zonas de Europa. Y sugieren evaluar el impacto de los episodios africanos sobre la salud humana y establecer sistemas adecuados de vigilancia. Estudios recientes en otras zonas de España han encontrado posibles relaciones causa-efecto entre los episodios de polvo africano y salud ${ }^{20}$.

En el segundo de los artículos se evalúa el posible impacto sobre los niveles de $\mathrm{Pb}$, $\mathrm{Cd}, \mathrm{Cr}$ y $\mathrm{Hg}$ en población general adulta alrededor de una planta de valorización energética de residuos urbanos, que desde 2005 está en pleno funcionamiento en Alonsotegi (Bilbao). Los resultados muestran que no se observan diferencias de concentraciones en relación con la cercanía de la planta. Buena parte de la fuente del $\mathrm{Pb}$ continúa siendo laboral, mientras que la exposición al $\mathrm{Cr}, \mathrm{Cd}$ y $\mathrm{Hg}$ podría tener un origen alimentario. Esta situación refleja un escenario común en muchas regiones industriales de Europa. La presión para reducir los niveles de emisión de metales de la industria ha sido grande en muchas zonas europeas, y como consecuencia los niveles en aire ambiente y los de depósito atmosférico se han venido reduciendo en muchas zonas. Además, la prohibición de gasolina con $\mathrm{Pb}$ ha reducido los niveles de este metal 
en aire ambiente desde centenares de microgramos por metro cúbico a algunas pocas decenas.

Entre los metales que generan preocupación por su exposición ambiental y que están regulados por Directivas de calidad del aire destacan ${ }^{9,10} \mathrm{As}, \mathrm{Cd}, \mathrm{Hg}, \mathrm{Ni}$ y $\mathrm{Pb}$. Además de estos la OMS propone valores guía para Mn y V, y destaca el posible impacto sobre la salud humana del Cr. Aunque como exponíamos hay una tendencia a la baja en cuanto a exposición de determinados metales, se ha de resaltar la existencia de un nuevo escenario relacionado con el tráfico rodado. El incremento del parque de vehículos en ciudades fuertemente congestionadas por tráfico y la reducida precipitación atmosférica causan la acumulación de metales sobre el firme de rodadura. Estos provienen de los productos de abrasión de frenos, discos, ruedas y el propio firme. Aunque se hayan eliminado los asbestos en las pastillas de frenos, metales como $\mathrm{Fe}$, $\mathrm{Mn}, \mathrm{Cu}, \mathrm{Zn}, \mathrm{Sb}, \mathrm{Ba}, \mathrm{Zr}$, entre otros, son componentes abundantes en ellas y $\mathrm{Zn}$ en las ruedas. Una diferencia de los escenarios industriales respecto al descrito para el tráfico es que en este último caso la emisión se produce en aglomeraciones urbanas densamente pobladas. La solución para reducir este problema radica esencialmente en la disminución del flujo de vehículos en zonas urbanas.

\section{CONSIDERACIONES FINALES}

Muchos procesos o actividades emiten partículas, y muchos de ellos tienen lugar en aglomeraciones urbanas, con el consiguiente impacto directo sobre la población. Debido a ello, en las últimas décadas se ha hecho un gran esfuerzo para reducir las emisiones de procesos industriales, vehículos, generación eléctrica, emisiones domésticas y agrícolas. Sin embargo la mejora de la calidad de vida de los ciudadanos que evoluciona de manera paralela al desarrollo industrial y social, exige cada vez más tecnología para minimizar los efectos adversos de los contaminantes en la salud humana, en los ecosistemas y en bienes de naturaleza diversa. Ello es debido a que incluso los niveles alcanzados en la actualidad (muy inferiores a los de hace 30 años) tienen efectos adversos. En este escenario, la mejora de calidad del aire en el futuro en países plenamente desarrollados se ha de basar en fijar objetivos de calidad del aire muy estrictos para que de esta manera su cumplimiento exija el perfeccionamiento de la tecnología ambiental o la aplicación de planes y estrategias que permitan alcanzarlos. Esta situación es compleja dado que requiere de conocimientos científico-técnicos profundos, no siempre plenamente desarrollados cuando se aplican planes y programas. Ello puede crear situaciones en las que, en regiones con niveles de exposición relativamente bajos, a pesar de aplicar planes y programas para reducir aún más las emisiones, sus efectos no son siempre patentes en la reducción de la exposición ${ }^{11}$. La investigación futura en este campo se ha de enfocar a relacionar la reducción de emisiones y sus efectos en la reducción de la exposición.

Este escenario sin embargo no es aplicable más que en países plenamente desarrollados. Una gran parte de la población del planeta está expuesta a niveles de contaminantes equivalentes a los que la población de los países plenamente desarrollados estaba expuesta hace 30 años. En estas zonas el crecimiento de la población y un desarrollo poco sostenible, han causado un deterioro de la calidad del aire y el crecimiento continuado de las emisiones de contaminantes con incidencia en el clima global y en la salud de sus habitantes. El apoyo a estos países para que compatibilicen el desarrollo económico y social con una minimización de las emisiones atmosféricas, es básico para cualquier planificación en lo referente a emisiones de contaminantes con efectos adversos en la salud, e 
impacto en los ecosistemas y en la modificación del clima.

\section{AGRADECIMIENTOS}

A mis compañeros A Alastruey, T Moreno y M Viana, por sus sugerencias sobre el artículo.

\section{BIBLIOGRAFÍA}

1. WHO, 2006. Health risks of particulate matter from long-range transboundary air pollution. Joint WHO/ Convention Task force on the Health Aspects of Air Pollution, 2006. European Centre for Environment and Health Bonn Office; 2006. Disponible en: http://www.euro.who.int/document/E88189.pdf.

2. IPCC. Climate Change 2007: The Physical Science Basis. Contribution of Working Group I to the Fourth Assessment Report of the IPCC 978 052170596-7 Paperback. IPCC; 2007.

3. Pope, C.A., Dockery, D.W., 2006. Health effects of fine particulate air pollution: lines that connect. J Air Waste Manage Assoc. 2006; 56(6), 709-42.

4. Ballester F., Saez M., Perez S., Iñiguez C., Gandarillas A., Tobias A., Bellido J., Taracido M, et al. The EMECAM project, a multicentre study on air pollution and mortality in Spain. Combined results fro particulates and sulphur dioxide. Occup Environ Med. 2002, 59, 300-308.

5. Katsouyanni K, Touloumi G., Samoli E., Gryparis A., Le Tertre A., Monopolis Y., Rossi G., Zmirou D., Balleste r F., Boumghar A., Anderson H.R., Wojtyniak B., Paldy A., Braunstein R.., Pekkanen J. Schindler Ch., Schwartz J. Confounding and Effect Modification in the Short-Term Effects of Ambient Particles on Total Mortality: Results from 29 European Cities within the APHEA2 Project. Epidemiology. 2001; 12, 5, 521-531.

6. Künzli N, Kaiser R, Medina S, Studnicka M, Chanel O, Filliger P, Herry M, Horak F Jr, Puybonnieux-Texier V, Quénel P, Schneider J, Seethaler R, Vergnaud JC, Sommer H.,. Public-health impact of outdoor and traffic-related air pollution: a European assessment. Lancet. 2000; 356: 795 801.

7. Sunyer J, Castellsagué J, Sáez M, Tobias A, Antó JM. Air pollution and mortality in Barcelona. J Epidemiol Community Health. 1996; 50, 1, 76-80.
8. Logan WDP. Mortality in the London fog incident. Lancet. 1953; 1:336-338.

9. EC (European Commission). Council Directive 2004/107/EC of the European Parliament and of the Council of 15 December 2004 relating to arsenic, cadmium, mercury, nickel and polycyclic aromatic hydrocarbons in ambient air. Brussels: Off $\mathbf{J}$ Eur Communities L Legis 2004;23: 3-16.

10. EC (European Commission). Council Directive 2008/50/EC of the European Parliament and of the Council of 21 May 2008 on ambient air quality and cleaner air for Europe. Brussels: Off J Eur Communities L Legis. 2008; 152: 1-44.

11. Harrison RM, Stedman J, Derwent D. New Directions: Why are PM10 concentrations in Europe not falling? Atmos Environ. 2008; 42, 3: 603-606.

12. Wichmann H.E., Spix C., Tuch T., Wölke G., Peters A., Heinrich J., Kreyling W.G. \& Heyder J. Daily Mortality and Fine and Ultrafine Particles in Erfurt, Germany. Part I: Role of Particle Number and Particle Mass. Health Effects Institute; 98 .

13. Pérez N., Pey J., Querol X., Alastuey A., López J.M. \& Viana M. Partitioning of major and trace components in PM10-PM2.5-PM1 at an urban site in Southern Europe. Atmos Environ. 2008; 42: 1677-91.

14. Querol, X., A. Alastuey, A. Alastuey, T. Moreno, M.M. Viana, S. Castillo, J. Pey, S. Rodríguez, B. Artiñano, P. Salvador, M. Sánchez, S. Garcia Dos Santos, M.D. Herce Garraleta, R. FernandezPatier, S. Moreno-Grau, L. Negral, M.C. Minguillón, E. Monfort, M.J. Sanz, R. Palomo-Marín, E. Pinilla-Gil, et al. Spatial and temporal variations in airborne particulate matter (PM10 and PM2.5) across Spain 1999-2005. Atmos Environ. 2008; 42: 3964-79.

15. Lopez Villarrubia, García Pérez M.D., Petral N., Ballester F., Iñiguez C., Pita M.L. Caracterización del ambiente atmosférico de las Palmas de Gran Canaria y Santa Cruz de Tenerife. Rev Esp Salud Pública. 2008; 82: 439-508.

16. Zubeiro M.B., Aurrekoetxea J.J., Ibarlueza J.M., Arenaza M.J., Bastarretxea M., Rodríguez C., Sáenz J.R. Metales pesados ( $\mathrm{Pb}, \mathrm{Cd}, \mathrm{Cr}$ y $\mathrm{Hg}$ ) en población general adulta próxima a una planta de tratamiento de residuos urbanos de Bizkaia. Rev Esp Salud Pública. 2008; 82: 481-492.

17. Viana M., Querol X., Alastuey A., Cuevas E., Rodríguez S. Influence of African dust on the 
levels of atmospheric particulates in the Canary Islands air quality network. Atmos Environ. 2002; 36: 5861-75.

18. Alonso-Pérez S., Cuevas E., Querol X., Viana M., Guerra J.C. Impact of the Saharan dust outbreaks on the ambient levels of total suspended particles (TSP) in the marine boundary layer (MBL) of the Subtropical Eastern North Atlantic Ocean Atmos Environ. 2007;41: 9468-80.
19. Escudero, M., Querol, X., Ávila, A., Cuevas, E. Origin of the exceedances of the European daily PM limit value in regional background areas of Spain. Atmospheric Environment. 2007 41, 730-744.

20. Pérez L, Tobias A, Querol X, Alastuey A, Viana M, Pey J, González-Cabré M, Valero N, Künzli N, Sunyer S. Coarse particles from Saharan dust and daily mortality. Epidemiology. 2008. DOI: 10.1097/EDE.0b013e31818131cf. 2008. 